\title{
Optimal Planning of Distributed Energy Storage Systems in Active Distribution Networks Embedding Grid Reconfiguration
}

\author{
Mostafa Nick, Rachid Cherkaoui, Senior Member, IEEE, and Mario Paolone, Senior Member, IEEE
}

\begin{abstract}
In this paper we present a procedure for the optimal siting and sizing of Energy Storage Systems (ESSs) owned, and directly controlled by network operators of Active Distribution Networks (ADNs). The peculiarity of the proposed planning procedure consists in embedding the grid reconfiguration. We use a recently proposed conditionally exact convex Optimal Power Flow (OPF) as the core of the optimization model. We appropriately model the objective function to include both technical and economic aspects, while keeping the exactness of the relaxed convex OPF. In particular, the proposed procedure accounts for the minimization of: voltage-magnitude deviations, feeders'/lines' congestion, cost of supplying loads, and investment costs related to the ESSs. In addition, the seasonal configurations of the grid are determined based on (1) network security constraints, and (2) the minimum resistive losses. The stochasticity of loads and renewable productions also are taken into account. We suitably modeled the ESSs to consider their ability to support the network by both active and reactive powers. Two test cases are used to demonstrate, and quantify, the capabilities of the proposed procedure for providing optimal and feasible solutions.
\end{abstract}

Index Terms-Optimal power flow, energy storage systems, active distribution networks, resource planning, grid reconfiguration.

\section{NOMENCLATURE}

\begin{tabular}{|c|c|}
\hline \multicolumn{2}{|l|}{ Variables } \\
\hline$C_{l}$ & Energy reservoir capacity of ESS $l$ \\
\hline $\mathcal{C}_{s, \varphi}$ & $\begin{array}{l}\text { Objective function of scenario (subproblem) } \varphi \text { of } \\
\text { season } s\end{array}$ \\
\hline$d_{k l}\left(d_{l k}\right)$ & $\begin{array}{l}\text { The direction of the line between buses } k \text { and } l \\
\left(d_{k l}=1 \text { means the direction is from } k \text { to } l, d_{l k}=1\right. \\
\text { means the direction is from } l \text { to } k)\end{array}$ \\
\hline$E_{l}(t)$ & Energy stored in ESS $l$ at time $t$ \\
\hline$f_{l} / f_{k l}, \bar{f}$ & $\begin{array}{l}\text { Square of current producing losses in line } l \text { (line } \\
\text { between buses } k \text { and } l \text { ) } \\
\text { current auxiliary variable upper bound (see Fig. 1) }\end{array}$ \\
\hline$\hat{I}_{l}^{t}, \hat{I}_{l}^{b}$ & $\begin{array}{l}\text { Upper bound for square of current flows at top and } \\
\text { bottom of lines } l \text {, respectively }\end{array}$ \\
\hline$P_{n}^{E}, Q_{n}^{E}$ & $\begin{array}{l}\text { Active and reactive power output of ESS located at } \\
\text { bus } n\end{array}$ \\
\hline$Q_{l}^{s h}$ & $\begin{array}{l}\text { Reactive power injection associated with the shunt } \\
\text { capacitance of the lines connected to bus } l\end{array}$ \\
\hline $\mathcal{R}_{l}$ & Square of power rating of ESS $l$ \\
\hline $\begin{array}{l}S_{l}^{t}=P_{l}^{t}+j Q_{l}^{t} \\
\left(\hat{S}_{l}^{t}=P_{l}^{t}+j \hat{Q}_{l}^{t}\right. \\
\left.\bar{S}_{l}^{t}=\bar{P}_{l}^{t}+j Q_{l}^{t}\right)\end{array}$ & $\begin{array}{l}\text { Complex power flow entering line } l \text { from the top, } \\
\text { power auxiliary variables lower and upper bounds, } \\
\text { respectively (see Fig. 1) }\end{array}$ \\
\hline$s_{l}$ & Complex power absorbed at bus $l$ \\
\hline
\end{tabular}

The Authors are with École Polytechnique Fédérale de Lausanne (EPFL).

$\begin{array}{ll}S_{l}^{b}=P_{l}^{b}+j Q_{l}^{b}, & \text { Complex power flow entering bus } l \text { from the } \\ \hat{S}_{l}^{b}=\hat{P}_{l}^{b}+j \hat{Q}_{l}^{b}, & \text { bottom, corresponding auxiliary variables lower and } \\ \bar{S}_{l}^{b}=\bar{P}_{l}^{b}+j \bar{Q}_{l}^{b} & \text { upper bounds, respectively (see Fig. 1) } \\ S_{k l}\left(S_{l k}\right)= & \text { Complex power flow from bus } k \text { to } l \text { (from } l \text { to } k \text { ) } \\ P_{k l}\left(P_{l k}\right)+ & \\ j Q_{k l}\left(Q_{l k}\right) & \text { Binary variable associated with the presence of an } \\ u_{n} & \text { ESS at bus } n \\ v_{\text {up }(l)}, v_{l} & \text { Square of nodal voltage magnitude at bus } l \text { (up }(l) \text { ) } \\ \left(\hat{v}_{\text {up }(l)}, \hat{v}_{l}\right) & \text { voltage auxiliary variables of bus } l \\ \mathfrak{R}_{l}(t) & \text { Resistive losses of the ESS located at bus } l \text { at time } t \\ \chi_{k l} & \text { Binary variable associated with the switch between } \\ & \text { buses } k \text { and } l\end{array}$

Parameters

$b_{l}\left(b_{k l}\right) \quad$ Susceptance of line $l$ (between buses $k$ and $l$ )

$\mathfrak{B} \quad$ A big number

$C_{n}^{C}, C_{n}^{\mathcal{R}}, C_{n}^{f} \quad$ ESS investment costs (energy reservoir, power rating, fixed installation costs)

$C_{n}^{\max } \quad$ Maximum ESS energy reservoir capacity that can be installed at bus $n$

$\mathcal{E}^{u}, \mathcal{E}^{d} \quad$ Maximum and minimum allowed SoE of the ESS located at bus $l$, respectively

Total budget for ESS installation

$\mathcal{F}$

G

The adjacency matrix of the oriented graph of the grid

$I_{l}^{\max }\left(I_{k l}^{\max }\right) \quad$ Upper limit of line $l$ current-flow square (between buses $k$ and $l$ )

$N_{c}$ $P_{k l}^{\max }, Q_{k l}^{\max }$

Number of allowed cycles per day for a given ESSs

Upper limits of active and reactive powers of the

$\mathcal{R}_{n}^{\max } \quad$ Upper bound of the square of power rating capacity that can be installed at bus $n$

$t^{e} \quad$ Last time step of the day

$\Delta t \quad$ Time step length

$v_{l}^{\max } / v_{l}^{\min } \quad$ Upper and lower bounds associated with the bus $l$ voltage-magnitude square

$\mathcal{W}_{v}, \mathcal{W}_{I} \quad$ Weight coefficients associated with the voltage deviation and lines' congestion goals, respectively

$z_{l}=r_{l}+j x_{l} \quad$ Longitudinal impedance of line $l$ (between buses $k$ $\left(z_{k l}=r_{k l}+\quad\right.$ and $\left.l\right)$

$\left.j x_{k l}\right)$

$\xi(t)$

Energy price profile

$\pi_{s, \varphi} \quad$ probability of the scenario $s$ of $\varphi$

$\sigma \quad$ Annual interest rate

Sets and indices

$l \quad$ Index of lines and buses other than slack bus 


\begin{tabular}{|c|c|}
\hline $\mathcal{L}$ & Set of lines (buses except the slack one) \\
\hline $\mathcal{L}_{s}$ & Set of lines with switches \\
\hline $\mathcal{L} \backslash \mathcal{L}_{s}$ & Set of lines without switches \\
\hline$s$ & Index of seasons in the master problem \\
\hline $\mathcal{S}$ & Set of seasons in master problem \\
\hline$n$ & Index of buses \\
\hline $\mathcal{N}$ & Set of buses (for reconfiguration part) \\
\hline $\mathcal{N}_{G}$ & Set of substation buses (for reconfiguration part) \\
\hline $\mathcal{N} \backslash \mathcal{N}_{G}$ & Set of buses other than substations \\
\hline$y$ & Index of year \\
\hline$\Omega(\zeta, \theta)$ & $\begin{array}{l}\text { Set of equations associated with reconfiguration } \\
\text { model ( } \zeta \text { : vector of variable, } \theta \text { : vector of } \\
\text { parameters) }\end{array}$ \\
\hline$\Psi(\alpha, \beta)$ & $\begin{array}{l}\text { Set of equations associated with ESS model ( } \alpha \text { : } \\
\text { vector of variable, } \beta \text { : vector of parameters) }\end{array}$ \\
\hline$\Theta(\gamma, \tau)$ & $\begin{array}{l}\text { Set of equations associated with the Augmented } \\
\text { Relaxed Optimal Power Flow (AR-OPF) ( } \gamma \text { : vector } \\
\text { of variable, } \tau \text { : vector of parameters) }\end{array}$ \\
\hline$\varphi$ & Index of scenarios in subproblems \\
\hline$\Upsilon_{\mathrm{s}}$ & $\begin{array}{l}\text { Set of subproblem scenarios associated with season } \\
S \text { of master problem }\end{array}$ \\
\hline
\end{tabular}

\section{INTRODUCTION}

$\mathrm{T}_{\mathrm{h}}^{\mathrm{h}}$ he lack of direct controllability of the Distributed Generations (DGs) is the main hindrance to the increase of the renewable energy resources penetration in distribution networks. Energy Storage Systems (ESSs) owned by Distribution Network operators (DNOs) will have an important role in the Active Distribution Networks (ADNs), since they have the capability to provide both technical services and economic benefits (by providing both active and reactive powers) [1]- [4]. The ESSs optimal siting and sizing including the possible network reconfiguration is the first challenge to be addressed, which is the focus of this paper.

Optimal Power Flow (OPF) is the core of optimization problems for control, operation, and planning of power networks. The main and common technical drawback of the existing literature related to the resource planning in ADNs is associated with the appropriate formulation of the OPF with regard to its non-convexities. Recently, several works have been proposed to convexify the OPF e.g., [5]-[7]. In particular, Semi Definite Programming (SDP) and Second Order Cone Programming (SOCP) relaxations are used for this purpose. It is shown in [6] that the SOCP is more computationally efficient with reference to SDP. In this respect, we have employed the exact convex OPF model proposed in [7], since the lines' transverse-parameter as well as the network static operational constraints (lines ampacity limits and nodal voltage magnitudes) are appropriately included. Note that the exactness of the model proposed in [7] is conditional. The derived conditions in [7] are mild and hold for realistic distribution grids. In Section V, we show that these conditions also hold for the benchmark networks used in this paper.

An important aspect of ADN operation, which is neglected in the literature, is related to the inclusion of $\mathrm{ADN}$ reconfiguration in the targeted problem. As known, the distribution networks are designed in meshed structure but operated as radial one. Consequently, they can be operated with different topologies as function of the utilities requirements. This aspect influences the optimal planning problem of ESSs. In this paper, we have incorporated the network reconfiguration into the optimal ESS siting and sizing using a two-stage formulation. In the first-stage, the ESSs site and size as well as the network configurations are determined. The second stage evaluates the benefits of the allocated ESSs.

Furthermore, we have appropriately modified the objective function proposed in [3] in order to preserve the exactness of the SOCP relaxation used for the OPF. As stated in [6], and [7], the objective function of the OPF have to be strictly increasing in total resistive losses, in order to have an exact relaxed OPF model. In this respect, we have appropriately modified the terms associated with the minimization of the nodal over-voltages and lines' congestion using the auxiliary variables for nodal voltage-magnitude and lines' currentflow. In case of large reverse power flow (power flow toward the external grid), this permits to model the nodal overvoltages and lines' congestion minimization.

The solution of the targeted planning problem is computationally challenging due to the large-scale size and/or presence of discrete variables. The inherent large-scale nature of the problem lays on the fact that it should cover a reasonable number of scenarios in order to obtain a solution accounting for a sufficiently large set of variations of the considered parameters. In this respect, one of the most common approaches is to decompose the optimization problem. We followed this approach employing the Bender decomposition in order to breakdown the problem into a master one and a set of (convex) subproblems [8-10]. As a side note, it is worth observing that the convergence of the Benders decomposition is guaranteed if the subproblems are convex, which is the case here (see [8]-[9]).

In view of the above, the contributions of the paper are here summarized. The paper defines the most important factors affecting the problem of ESSs siting and sizing in ADNs; specifically, we have (i) incorporated the network reconfiguration, (ii) used a conditionally exact convex model of OPF, (iii) formulated the objective function of the OPF accounting for the grid technical operation objectives (voltage-magnitudes' deviation and lines' congestion) while preserving the exactness of the OPF relaxation, and (iv) applied the Benders decomposition for the solution of this specific optimization problem.

The structure of the paper is as the following: In Section II, we review the related works. In Section III, we present the structure of the optimization problem and its different parts. The problem formulation and solution methodology are described in Section IV. In Section V, we provide numerical evaluation of our method. Finally, Section VI concludes the paper.

\section{RELATED WORK}

A large literature exists concerning the optimal siting and sizing of the ESSs in ADNs. A comprehensive survey of this subject is presented in [11]. A cost-benefit analysis of ESSs for peak demand reduction in medium voltage grids is proposed in [12]. A number of works employ heuristic methods to solve the targeted optimization problem with 
single or multi-objective goals including voltage control, losses minimization, energy supply cost minimization, etc. (e.g., [2], and [13]- [18]). In [15] it is presented a cost-benefit analysis of the use of ESSs for peak demand reduction in medium-voltage distribution networks. The objective function considers (i) savings in energy costs derived from storing energy at low-priced hours and selling it at peak hours and (ii) savings in network reinforcement thanks to the peak shaving. In [17] the Authors use the non-dominated sorting genetic algorithm for the solution of optimal allocation of DGs in distribution networks considering off-line network reconfiguration. The objective function minimizes resistive losses, energy not supplied (ENS), and the costs associated with DGs. In [19] a procedure is proposed for siting and sizing of storage devices in order to support both transmission and distribution networks. The objective function includes provision of frequency and reserve services in transmission network; peak shaving and load curve smoothing in distribution networks. A cost-benefit approach is presented in [20] to find the optimal sites and sizes of ESSs in distribution networks. The goal of the optimization is to maximize the DNO profits from energy transactions, as well as investment and operation costs savings. In [21] a two-stage methodology is proposed for optimal ESS sizing. The first stage determines initial storage sizes considering hourly wind and load profiles. The second stage adopts a mono-period bi-level AC OPF to tune the first-stage storage sizes considering lines congestion and voltages limits, on-load tap changers, DG power factor, and renewable energies curtailment.

The above-mentioned works either use the non-convex formulation of power flow equations or do not consider the full AC power flow equations. Recently, several works have used the convex formulations of AC-OPF for ESS planning in ADNs. The relaxed Second Order Cone Programming (SOCP) model of the OPF is used in [3], [22]-[23] in order to convexify the power flow equations. However, the relaxed OPF used in these works may face difficulties in case of reverse power flow [24]. The methodology presented in [22] first determines the size of the required ESS for mitigating the impacts of large-scale distributed PV. Afterward, the optimal locations for the ESS along the feeder, taking into account the AC power flow equations is determined. In [3] a multiobjective approach for siting and sizing of ESSs considering both technical and economic goals is presented. The work is further augmented in [23 by decomposing the optimization problem using Alternative Direction Method of Multiplies (ADMM) and make it applicable for large-scale case studies.

\section{PROBLEM STRUCTURE}

In what follows, we describe the different parts composing the proposed procedure for the targeted optimization problem. In particular, the load flow equations and auxiliary load flow equations, used to formulate the grid security constraints, are first described. Furthermore, the developed model of lossy ESSs, including the State-of-Energy ( $S o E)^{1}$, capability curve, and reservoir capacity constraints, is presented. Finally, the power flow equations with radiality constraints and the uncertainty modeling of the stochastic parameters (load, PV and energy prices) are given.

\section{A. Power flow and auxiliary power flow equations}

For a given radial power network, the power flow equations, considering the lines shunt impedances, are given in (1.a)-(1.d). For sake of clarity, and to introduce a part of nomenclature, the complete transmission line two-port $\Pi$ model is shown in Fig. 1.

$$
\begin{gathered}
S_{l}^{t}=s_{l}+\sum_{m \in \mathcal{L}} \mathbf{G}_{l, m} S_{m}^{t}+z_{l} f_{l}-j\left(v_{\mathrm{up}(l)}+v_{l}\right) b_{l}, \quad \forall l \in \mathcal{L} \\
v_{l}=v_{\mathrm{up}(l)}-2 \Re\left(\mathrm{z}_{l}^{*}\left(S_{l}^{t}+j v_{\mathrm{up}(l)} b_{l}\right)\right)+\left|z_{l}\right|^{2} f_{l}, \forall l \in \mathcal{L} \\
f_{l}=\frac{\left|S_{l}^{t}+j v_{\mathrm{up}(l)} b_{l}\right|^{2}}{v_{\mathrm{up}(l)}}=\frac{\left|S_{l}^{b}-j v_{l} b_{l}\right|^{2}}{v_{l}}, \quad \forall l \in \mathcal{L} \\
S_{l}^{b}=S_{l}+\sum_{m \in \mathcal{L}} \mathbf{G}_{l, m} S_{m}^{t}, \quad \forall l \in \mathcal{L}
\end{gathered}
$$

Where $\mathfrak{R}$ represents the real part of an imaginary number. Buses other than the slack bus are denoted with $1, \ldots, L ; \mathcal{L}$ denotes the set $\mathcal{L}=\{1,2, \ldots, L\}$ and $\operatorname{up}(l)$ is the label of the bus that is upstream of bus $l$. We also label with $l$ the line whose downstream bus is bus $l$; its upstream bus is therefore $\operatorname{up}(l) . \mathbf{G}$ is the adjacency matrix of the oriented graph of the network, i.e. $\mathbf{G}_{k, l}$ is defined for $k, l \in \mathcal{L}$ and $\mathbf{G}_{k, l}=1$ if $k=$ $\operatorname{up}(l)$ and 0 otherwise. $S_{l}^{t}=P_{l}^{t}+j Q_{l}^{t}$ is the complex power flow entering line $l$ from the top, i.e. from bus $\operatorname{up}(l) ; S_{l}^{b}=$ $P_{l}^{b}+j Q_{l}^{b}$ is the complex power flow entering bus $l$ from the bottom part of line $l, f_{l}$ is the square of the current in the central element of line $l$ (Fig. 1), $z_{l}=r_{l}+j x_{l}$ and $2 b_{l}$ are the longitudinal and shunt impedances of line $l$. We denote with $\mathrm{z}_{l}^{*}$ the complex conjugate of $z_{l}, v_{l}$ is the square of voltage magnitude and $s_{l}=p_{l}+j q_{l}$ is the power absorption at bus $l$ ( $p_{l} \geq 0$ and $q_{l} \geq 0$ denotes power consumptions, $p_{l} \leq 0$ and $q_{l} \leq 0$ denote powers injections).

Equations (1.a)-(1.c) are directly derived by the application of the Kirchhoff's law to Fig. 1 and represent the power and voltage equilibriums in each line. Equation (1.d) represents complex power flow of line $l$ at its $l$ side.

An optimization problem with the original power flow equations is non-convex due to equation (1.c). However, it becomes convex if we replace (1.c) by the following inequality:

$$
f_{l} v_{\text {up }(l)} \geq\left|S_{l}^{t}+j v_{\text {up }(l)} b_{l}\right|^{2}, \quad \forall l \in \mathcal{L}
$$

However, it may often occur that the optimal solution of the relaxed optimization problem (i.e., with (2)) does not satisfy the original constraint (1.c), (i.e. the obtained solution has no physical meaning [24]). In particular, this may happen when either one of the nodal voltage upper-bounds or lines ampacity limit is/are binding. In [7] the Authors propose a way to modify the relaxed OPF to ensure the exactness of the relaxation. In particular, they propose to use auxiliary

\footnotetext{
${ }^{1}$ We use the term state-of-energy $(S o E)$ to indicate the energy, in per unit, accumulated in a given ESS.
} 


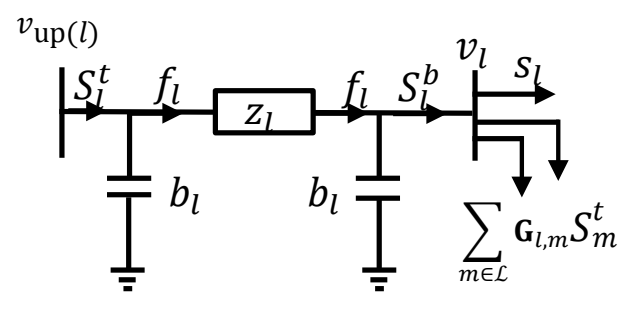

Fig. 1: Classical two-port $\Pi$ model of a transmission line adopted for the formulation of the OPF relaxed constraints.

variables in order to formulate the grid security constraints. The main idea of modifying the OPF problem is to account for the security constraints using a set of auxiliary variables that: i) are upper bounding the nodal voltage magnitudes and line current magnitudes and ii) do not depend on $f$. In this respect, it is introduced a new set of auxiliary variables: $\bar{f}, \hat{S}$ , $\bar{S}$ for the lines of the grid and $\hat{v}$ for the buses of the network as defined in (3.a)- (3.f). $\hat{S}$ and $\hat{v}$ represent lower bound and upper bound on $S$ and $v$, respectively whereas $\bar{S}$ and $\bar{f}$ are upper bounds on $S$ and $f$, respectively (see [7] for further details). Note that the exactness of the model proposed in [7] is conditional and, as already mentioned, the conditions are mild and hold for realistic distribution networks. These conditions imply that, when both active and reactive power losses of one or more than one lines decrease, we have: (i) the increase of voltage magnitude in all buses, (ii) the net active power imported (exported) from (to) the external transmission network decreases (increases), and (iii) the procedure to obtain a new load flow solution with the reduced losses converges.

$$
\begin{gathered}
\hat{S}_{l}^{t}=s_{l}+\sum_{l \in \mathcal{L}} \mathbf{G}_{l, m} \hat{S}_{l}^{t}-j\left(\hat{v}_{\mathrm{up}(l)}+\hat{v}_{l}\right) b_{l}, \quad \forall l \in \mathcal{L} \\
\hat{v}_{l}=\hat{v}_{\mathrm{up}(l)}-2 \Re\left(\mathrm{z}_{l}^{*}\left(\hat{S}_{l}^{t}+j \hat{v}_{\mathrm{up}(l)} b_{l}\right)\right), \quad \forall l \in \mathcal{L} \\
\bar{S}_{l}^{t}=s_{l}+\sum_{m \in \mathcal{L}} \mathbf{G}_{l, m} \bar{S}_{m}^{t}+z_{l} \bar{f}_{l}-j\left(v_{\mathrm{up}(l)}+v_{l}\right) b_{l}, \quad \forall l \in \mathcal{L} \\
\bar{f}_{l} v_{l} \geq\left|\max \left\{\left|\hat{P}_{l}^{b}\right|,\left|\bar{P}_{l}^{b}\right|\right\}\right|^{2}+\left(\max \left\{\left|\hat{Q}_{l}^{b}-j \hat{v}_{l} b_{l}\right|,\left|\bar{Q}_{l}^{b}-v_{l} b_{l}\right|\right\}\right)^{2}, \\
\forall l \in \mathcal{L} \\
\bar{f}_{l} v_{\mathrm{up}(l)} \geq\left|\max \left\{\left|\hat{P}_{l}^{t}\right|,\left|\bar{P}_{l}^{t}\right|\right\}\right|^{2} \\
+\left(\operatorname { m a x } \left\{\left|\hat{Q}_{l}^{t}+j \hat{v}_{\mathrm{up}(l)} b_{l}\right|, \mid \bar{Q}_{l}^{t}\right.\right. \\
\left.\left.+v_{\mathrm{up}(l)} b_{l} \mid\right\}\right)^{2}, \forall l \in \mathcal{L} \\
\bar{S}_{l}^{b}=s_{l}+\sum_{m \in \mathcal{L}} \mathbf{G}_{l, m} \bar{S}_{l}^{t}, \quad \forall l \in \mathcal{L}
\end{gathered}
$$

The grid static security constraints composed by: (i) the nodal voltage magnitudes and (ii) lines ampacity limit constraints, can be formulated as follows (for further details see [7]).

$$
v^{\min } \leq v_{l}, \quad \forall l \in \mathcal{L}
$$

$$
\begin{aligned}
& \hat{v}_{l} \leq v^{\max }, \quad \forall l \in \mathcal{L} \\
& || \max \left\{\left|\hat{P}_{l}^{b}\right|,\left|\bar{P}_{l}^{b}\right|\right\}\left|+j \max \left\{\left|\hat{Q}_{l}^{b}\right|,\left|\bar{Q}_{l}^{b}\right|\right\}\right|^{2} \leq v_{l} \hat{I}_{l}^{b} \leq v_{l} I_{l}^{\max }, \\
& \mid \max \left\{\left|\hat{P}_{l}^{t}\right|,\left|\bar{P}_{l}^{t}\right|\right\}+j\left(\left.\max \left\{\left|\hat{Q}_{l}^{t}\right|,\left|\bar{Q}_{l}^{t}\right|\right\}\right|^{2} \leq v_{\text {up }(l)} \hat{I}_{l}^{t}\right. \\
& \leq v_{\text {up }(l)} I_{l}^{\max }, \forall l \in \mathcal{L}
\end{aligned}
$$

where $v_{l}^{\max } / v_{l}^{\min }$ is upper/lower limit of the bus $l$ voltage magnitudes' square and and $I_{l}^{\max }$ is the upper limit of the square of current flow of the line $l$.

For the sake of brevity, hereinafter, the set of abovementioned equations ((1.a), (1.b), (1.d), (3), (4)) are represented as follows:

$$
\Theta(\gamma, \tau) \geq 0
$$

where $\quad \gamma=\left\{S^{t}=P^{t}+j Q^{t}, v, f, \hat{S}^{t}=\hat{P}^{t}+j \hat{Q}^{t}, \hat{v}, \bar{f}, \bar{S}^{t}=\right.$ $\left.\bar{P}^{t}+j \bar{Q}^{t}, s, \hat{I}^{b}, \hat{I}^{t}\right\}$ is the set of variables and $\tau=$ $\left\{z, b, v_{l}^{\max }, v_{l}^{\min }, I^{\max }\right\}$ is the set of parameters. The notation without subscript represents the vector of corresponding variable/parameter for the all buses/lines.

\section{B. Energy storage systems}

We model the ESS charging/discharging losses with its equivalent resistance connecting an ideal ESS to the grid ${ }^{2}$. In this respect, we connect each ESS through a new line whose impedance is equal to the ESS equivalent resistance (see Fig. 2).

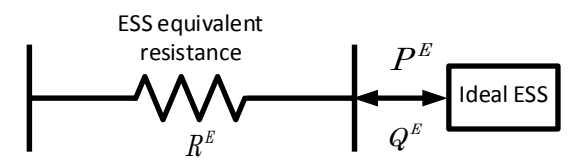

Fig. 2: ESS model

The ideal ESS is modeled with equations (6.a) - (6.d).

$$
\begin{gathered}
E(t)=E(t-1)-P^{E}(t) \Delta(t)-\mathfrak{L}(t) \Delta(t) \\
C \mathcal{E}^{d} \leq E(t) \leq C \mathcal{E}^{u} \\
\left(Q^{E}(t)\right)^{2}+\left(P^{E}(t)\right)^{2} \leq \mathcal{R}^{2} \\
E(1)=E\left(t^{e}\right) \\
\frac{\Delta t}{2 * 3600}\left|\omega P^{E}(t)\right| \leq N_{c} C \\
\mathfrak{L}(t) \geq r^{E}\left(\left(Q^{E}(t)\right)^{2}+\left(P^{E}(t)\right)^{2}\right)
\end{gathered}
$$

where $E(t)$ is the energy stored in the ESS at time step $t$, $P^{E}(t)$ and $Q^{E}(t)$ are the active and reactive power outputs of the ESS at time $t, \mathfrak{L}(t)$ represents the resistive losses in the battery energy storage, $\Delta t$ is the time step length, $\mathcal{E}^{l}$ and $\mathcal{E}^{u}$ are the maximum and minimum allowed $S o E$ levels of the ESS. $C$ and $\mathcal{R}$ are the reservoir capacity and square of power

\footnotetext{
2 The value of the resistance can be inferred from a fitting problem representing the ESS losses provided by the model of Fig. 2 and the real ones at different discharge rates and $S o E$.
} 
rating of the ESS. $N_{c}$ is the allowed number of cycles per day chosen as a function of the desired end-of-life time of the ESS (for instance, 20 years) (see [25] for further details in case ESS is a battery). Finally, $t^{e}$ is the last time step of the day.

Equation (6.a) represents the $S o E$ equation of the ESS. It implies that the amount of stored energy in the ESS reservoir at time $t$ depends on its $S o E$ in the previous time step and the net energy injected/extracted from it. The maximum and minimum SoE limits are modeled by equation (6.b). ESSs are normally interfaced with the grid using a power electronic converter. In this respect, their capability curve (on the AC side of the grid interface) is governed by the ampacity limit of the power converter that, in case of an operation under constant AC grid voltage, can be translated into a constraint to the apparent power delivered by the ESS (6.c). Equation (6.d) implies that the energy stored in the ESSs at the end of the day have to be identical to its initial value. In case the ESS is composed by a battery, we account for the degradation of the battery cells using equation (6.e) (this constraint is defined using the approach proposed in [25]). More specifically, this constraint, bounds the exchanged energy of the battery. Note that $\omega$ is a positive parameter and its value depends on $P^{E}(t)$. We chose the maximum value of $\omega$ using the indications provided in [25] $(\omega=1)$. Finally, constraint (6.f) models the ESS resistive losses.

For the sake of brevity, hereinafter in this paper, the set of above-mentioned equations (ESS model) are represented as follows:

$$
\Psi(\alpha, \beta) \geq 0
$$

where $\alpha=\left\{P^{E}, Q^{E}, E, \mathcal{R}, C, \mathfrak{L}\right\}$ is the set of variables and $\beta=\left\{\Delta(t), \omega, N_{c}, \mathcal{E}^{u}, \mathcal{E}^{d}\right\}$ is the set of parameters. The notation without subscript represents the vector of corresponding variable/parameter for the all buses/lines.

\section{Power flow equations with radiality constraints}

The radiality constraints are modeled with the set of equations (8.a) - (8.e). We have employed the radiality constraints proposed in [26]. The main idea is to ensure that every single bus is supplied by only one substation through a unique path. In other words, any loop that includes one or more substations is not a feasible solution. The loops without substations cannot supply the loads, thus they are unfeasible solutions. The variable $d_{k l}$ defines the direction between buses $k$ and $l\left(d_{k l}=1\right.$ means the direction is from $k$ to $l$ and $d_{l k}=1$ means the direction is from $l$ to $k$ ). Note that these are continuous variables. Binary variables are used for the lines with a switch. However, the identified values of $d_{k l}$ or $d_{l k}$ are either 0 or 1 (see [26] for the proof of this property). The adoption of this modeling approach allows the optimization problem to have less binary variables as they are only used for the lines with a switch. $\mathcal{L}$ is the set of the lines. $\mathcal{L}_{s}$ is the set of the lines with switches whereas $\mathcal{L} \backslash \mathcal{L}_{s}$ represents the set of the lines without switch. $\mathcal{L}_{l}$ represents the set of the lines connected to bus $l$. The set of all buses is represented by $\mathcal{N}$. The buses that are substations are represented by $\mathcal{N}_{G}$ whereas the set of remaining buses is represented by $\mathcal{N} \backslash \mathcal{N}_{G}$. The constraint (8.a) implies that the lines without switch must have only one direction (either from $k$ to $l$ or from $l$ to $k$ ). Every switch has one direction in case its switch $\left(\chi_{k l}\right)$ is on. This is modeled by equation (8.b). The direction of any line connected to a substation is from the substation to its corresponding adjacent bus. It is shown in the equation (8.c). The buses that are not substation can only have one infeed as modeled by (8.d).

$$
\begin{gathered}
d_{k l}+d_{l k}=1, \forall(l, k) \in \mathcal{L} \backslash \mathcal{L}_{s} \\
d_{k l}+d_{l k}=\chi_{k l}, \quad \forall(l, k) \in \mathcal{L}_{s} \\
d_{k l}=0, \quad \forall l \in \mathcal{N}_{G} \\
\sum_{k:}\left(d_{k l}\right)=1, \quad l \in \mathcal{N} \backslash \mathcal{N}_{G} \\
\chi_{k l} \in\{0,1\}, \forall(l, k) \in \mathcal{L}_{s}
\end{gathered}
$$

Using these direction variables, the power flow equations, are modeled as shown in (9.a)-(9.h). Note that the linear DistFlow model is used here [27]. We appropriately modified the DistFlow model to incorporate the shunt elements of the lines.

$$
\begin{gathered}
-d_{k l} P_{k l}^{\max } \leq P_{k l} \leq d_{k l} P_{k l}^{\max }, \forall(l, k) \in \mathcal{L} \\
-d_{l k} P_{l k}^{\max } \leq P_{l k} \leq d_{l k} P_{l k}^{\max }, \forall(l, k) \in \mathcal{L} \\
-d_{k l} Q_{k l}^{\max } \leq Q_{k l} \leq d_{k l} Q_{k l}^{\max }, \forall(l, k) \in \mathcal{L} \\
-d_{l k} Q_{l k}^{\max } \leq Q_{l k} \leq d_{l k} Q_{l k}^{\max }, \forall(l, k) \in \mathcal{L} \\
v_{k} \leq v_{l}-2 \Re\left(z_{k l}^{*} S_{k l}\right)+\mathfrak{B}\left(1-d_{k l}\right), \forall l \in \mathcal{N} \backslash \mathcal{N}_{G} \\
v_{k} \geq v_{l}-2 \Re\left(z_{k l}^{*} S_{k l}\right)-\mathfrak{B}\left(1-d_{k l}\right), \forall l \in \mathcal{N} \backslash \mathcal{N}_{G} \\
v_{l} \leq v_{k}-2 \Re\left(z_{k l}^{*} S_{l k}\right)+\mathfrak{B}\left(1-d_{l k}\right), \forall l \in \mathcal{N} \backslash \mathcal{N}_{G} \\
v_{l} \geq v_{k}-2 \Re\left(z_{k l}^{*} S_{l k}\right)-\mathfrak{B}\left(1-d_{l k}\right), \forall l \in \mathcal{N} \backslash \mathcal{N}_{G} \\
\sum_{k:(l, k) \in \mathcal{L}_{l}}\left(S_{l k}-S_{k l}\right)+s_{l}=j Q_{l}^{s h}, \forall l \in \mathcal{N} \\
v^{\min } \leq v_{k} \leq v^{\max }, \forall l \in \mathcal{N} \backslash \mathcal{N}_{G} \\
\left(P_{k l}\right)^{2}+\left(Q_{k l}-v_{k} b_{k l}\right)^{2} \leq v^{\min } I_{l}^{\max }, \forall(l, k) \in \mathcal{L} \\
\left(P_{l k}\right)^{2}+\left(Q_{l k}-v_{l} b_{k l}\right)^{2} \leq v^{\min } I_{l}^{\max }, \forall(l, k) \in \mathcal{L} \\
Q_{l}^{s h}=\sum_{k:(l, k) \in \mathcal{L}_{l}}\left(b_{k l} v_{l}\right), \forall l \in \mathcal{N} \backslash \mathcal{N}_{G}
\end{gathered}
$$

where $S_{k l}=P_{k l}+j Q_{k l}\left(S_{l k}=P_{l k}+j Q_{l k}\right)$ is the complex power flow from bus $k$ to $l$ ( $l$ to $k$ ) after the shunt element of the line (in the central part of the line). $Q_{l}^{s h}$ is the reactive power associated with the shunt elements of the lines connected to bus $l$. $\mathfrak{R}$ represents the real part of an imaginary number.

Constraints (9.a) and (9.b) impose the flow limits based on the lines' direction variables $\left(d_{k l}, d_{l k}\right)$. The direction of the line could be either from $k$ to $l$ or vice versa. It should be noted that with any direction, the active and reactive power flows could have both positive and negative values. The voltage drop across the line is modeled by equations (9.c) and (9.d). The big number approach ensure that these constraints are always satisfied for open switches. For the closed switches and other lines, this constraint is always satisfied either for $k$ to $l$, or for $l$ to $k . \mathfrak{B}$ is a big number (in per unit system 3 is big enough). The nodal power balance equation is 
modeled in (9.e). The nodal voltage magnitudes' upper and lower limits and the lines ampacity constraints are modeled using equations (9.f)- (9.h). It should be noted that in equations (9.g) and (9.h) the reactive powers associated to the shunt elements of the lines are added to the lines current flow.

For the sake of brevity, hereinafter in this paper, the set of above-mentioned equations (radiality constraints and load flow equations with radiality constraints) are represented as follows:

$$
\Omega(\zeta, \theta) \geq 0
$$

where $\zeta=\{v, S=P+j Q, d, \chi, s\}$ is the set of variables and $\theta=\left\{v^{\text {min }}, I^{\text {max }}, v^{\text {max }}, z, \mathfrak{B}\right\}$ is the set of parameters.

\section{Uncertain Parameters}

The parameters (load, PV, and energy price) of the optimization problem have uncertainties. These variations are characterized by their stochastic behaviors over different time spans. The PV profiles have variations across the seasons and they have dissimilar characteristics for the cloudy and clear sky days. The load and energy prices have seasonal variations in addition to dissimilarities in weekdays and weekends. Additionally, their profile can have variations with respect to the external parameters like temperature and holidays. In this paper, we have considered all these variations in order to generate scenarios. The scenario generation and reduction are explained in the following sub-sections.

\section{1) Scenario generation}

The historical data of the parameters (load, solar irradiation) are assumed to be available. These data are grouped with different criteria. In case of load and energy price, the profiles are first divided into 4 bins representing the seasonal variations (winter/spring/fall/summer). The grouped data for each season is again divided in two categories: weekdays, weekends. Finally, the data in the weekends and weekdays bins are further divided into two groups. This last step is necessary since there may exist a big variation in the weekend/weekdays load/energy price profiles of each season with respect to temperature or other external factors. Similarly, the PV data is distributed between 4 groups representing the seasonal variations. The data of each season is again divided with respect to the irradiation profiles for clear/cloudy or partially cloudy sky.

In order to generate scenarios, we have accounted for the temporal correlation of the parameters during the day. We assumed that the daily vector of each input parameter (load, and PV) has a multivariate Gaussian distribution with mean $\mu$ and covariance $\Omega(\mathcal{N}(\mu, \Omega))$. The mean and covariance matrix of the parameters are obtained empirically using the historical data in each bin. The obtained distributions are used to generate appropriate scenarios using multivariate normal random numbers for each bin. Once the scenarios for load and $\mathrm{PV}$ are generated, they are used to generate the final scenario tree $^{3}$.

The obtained scenarios for the PV production are distributed between the PV sites in proportion to their nominal power. In this way, we achieve their spatial correlation. In order to account for the spatial correlation of

3 The choice of using Gaussian distribution is driven by sake of reproducibility and goes beyond the scope of the paper. Indeed, since the the load data, the generated scenarios for the load are distributed between the buses of the system in proportion to their medium to low voltage transformer rating. The annual increase rates of the load and PV are considered to be constant (any other complex growth rate can be easily considered).

In case of energy price scenarios, the historical energy price data for one year are used as the initial scenarios. The energy and fuel prices' growth over the years are modeled by using the Geometric Brownian Motion (GBM) [28]. Algorithm 1 shows the scenario generation procedure.

\section{2) Scenario reduction}

Accounting for all possible scenarios may results in computationally expensive simulations. Thus, often the number of scenarios is reduced to a reasonable one characterized by the same degree of volatility/stochasticity of the original scenarios. The scenarios for our targeted problems are the daily load/PV/price ones. In other words, we have scenarios in the form of time series with $T_{L}+T_{P V}+T_{P r}$ dimension. $T_{L}, T_{P V}$ and , $T_{p r}$ are the length of the daily load, $\mathrm{PV}$, and price profiles respectively. The first step is to determine an appropriate criterion for comparing the resemblances of the scenarios. We have employed Euclidian distance to determine the similarities between each pair of daily profiles.

Once the similarities between every pair of the daily time series are determined, they are grouped into a binary hierarchical cluster tree. In this respect, the pairs of the objects are linked based on their proximity. The new formed clusters are again grouped into larger clusters and this continues until a hierarchical tree is formed. The distance between the newly formed clusters can be defined using various algorithms; here we have used the furthest distance of the two grouped scenarios.

The next step is to partition the data into the clusters using the created hierarchical tree of binary clusters. This can be done using several approaches. The simplest one is to determine the number of clusters by the user based on his intuition. It can be done also based on the clusters' inconsistency criteria or distances. Here we have selected the final number of clusters based on a predefined maximum distance. Finally, we have used K-Medoids algorithm to select one representative scenarios for each bin [29]. Algorithm 2 shows the scenario generation procedure.

\section{PROBlem Formulation}

The objective is to optimally determine the seasonal network configurations and global ESSs site and size in order to (i) decrease nodal voltage-magnitude deviations, (ii) lines' congestion, (iii) energy supply cost, and (iv) ESS investment costs. Our working hypothesis is to have one configuration per season. For each season, we take into account a number of scenarios to evaluate the operational benefits of ESSs. In this respect, the targeted optimization problem is shown in (11).

$$
\underset{\lambda, \mu, \varrho}{\operatorname{minimize}}\left(c_{\lambda}^{T} \lambda+c_{\varrho}^{T} \varrho\right)+\sum_{\varphi \in \Phi}\left(\rho^{\varphi} c_{\mu_{\varphi}}^{T} \mu_{\varphi}\right)
$$


subject to:

$$
\begin{gathered}
\mathbf{A} \mu_{\varphi}+\mathbf{B} \lambda+\mathbf{C} \varrho=\mathbf{D}_{\varphi}, \quad \forall \varphi \in \Phi \\
\mathbf{E} \mu_{\varphi}+\mathbf{F} \lambda+\mathbf{G} \varrho \leq \mathbf{H}_{\varphi}, \quad \forall \varphi \in \Phi \\
\mu_{\varphi} \in \mathcal{K}_{\varphi} \\
\varrho \in\{0,1\}
\end{gathered}
$$

$\varrho$ represents the binary variables associated with the ESSs allocation and seasonal status of switches. $\lambda$ represents the continuous variables associated with the seasonal network reconfiguration and ESS installation. $\mu_{\varphi}$ is the vector of variables related to the subproblems (AR-OPF with ESS) for scenario $\varphi$. Finally, $\mathcal{K}_{\varphi}$ is a convex envelope. The first goal $\left(c_{\lambda}^{T} \lambda+c_{\varrho}^{T} \varrho\right)$ corresponds to the minimization of the ESS investment and network resistive losses. The second objective $\sum_{\varphi \in \Phi}\left(\rho^{\varphi} c_{\mu_{\varphi}}^{T} \mu_{\varphi}\right)$ aims at minimizing the daily operational-costs of the grid (the cost includes the technical and economic ones). The details of these objectives are provided in the Sections IV.A and IV.B. Constraints (11.b) and (11.c) corresponds to the ESS operation and installation costs, AR-OPF, and network reconfiguration. More details about these constraints are provided in Sections IV.A and IV.B.

We decompose the targeted optimization problem using an iterative two stages procedure. Normally, planning problems deal with large number of scenarios in order to cover all the possible future operating conditions of a given system. In this respect, the size of the problem could easily explode, especially for networks with a large number of nodes. We have applied Benders decomposition to break down the problem and decrease its computational complexity [8-10]. In this respect, the problem is decomposed to a master problem and several subproblems. The subproblems are independent of each other and can be solved simultaneously and in parallel. The first stage, called master problem, determines the site and size of the ESS units as well as the state of the switches (the network configuration) for each season. The fitness of the master problem solution is determined in the second stage, called subproblem. The convergence criteria is the difference between the Upper Bound, $U B=\mathcal{C}^{i *}+$ $\sum_{s \in \mathcal{S}} \sum_{\varphi \in \mathrm{Y}_{s}}\left(\mathcal{C}_{s, \varphi}^{*}\right)$ (see equations 11.a and (12.a), indicates that it is the identified optimal solution) and the Lower Bound, $L B=\mathcal{C}^{i *}+\mathcal{C}^{s *}$ (see equation (11.a), * indicates that it is the identified optimal solution). It should be less than a predefined value. The LB corresponds to the objective value of the master problem. The UB corresponds to the objective value of the subproblems plus the investment cost and resistive losses in the master problem. Note that the Master problem is a Mixed-Integer QuadraticallyConstrained Quadratic Programming (MIQCQP) and the subproblems are Second Order Cone Programming (SOCP). The Benders decomposition casted to our planning problem is shown in Algorithm 3.

\section{A. Master problem}

The ESS sites and sizes are determined in this stage.
Moreover for each season $(s \in \mathcal{S})$, the grid configuration is determined. We determine the network configuration with one daily profile with time step discretization of 2 hours (the time step discretization is 15 minutes in the subproblems). The main purpose of the switching in the master stage is to choose the feasible (satisfying the nodal voltage-magnitude and lines' ampacity limits) network configuration for each season. In this respect, we consider the worst daily profile for each season in the master problem. In winter and fall, we consider a daily profile with maximum load and minimum $\mathrm{PV}$; and in summer and spring, we consider a profile with maximum PV and minimum load. The price scenarios are not considered here, as they are assumed to not influence the network configuration. Note that no cost is assumed for

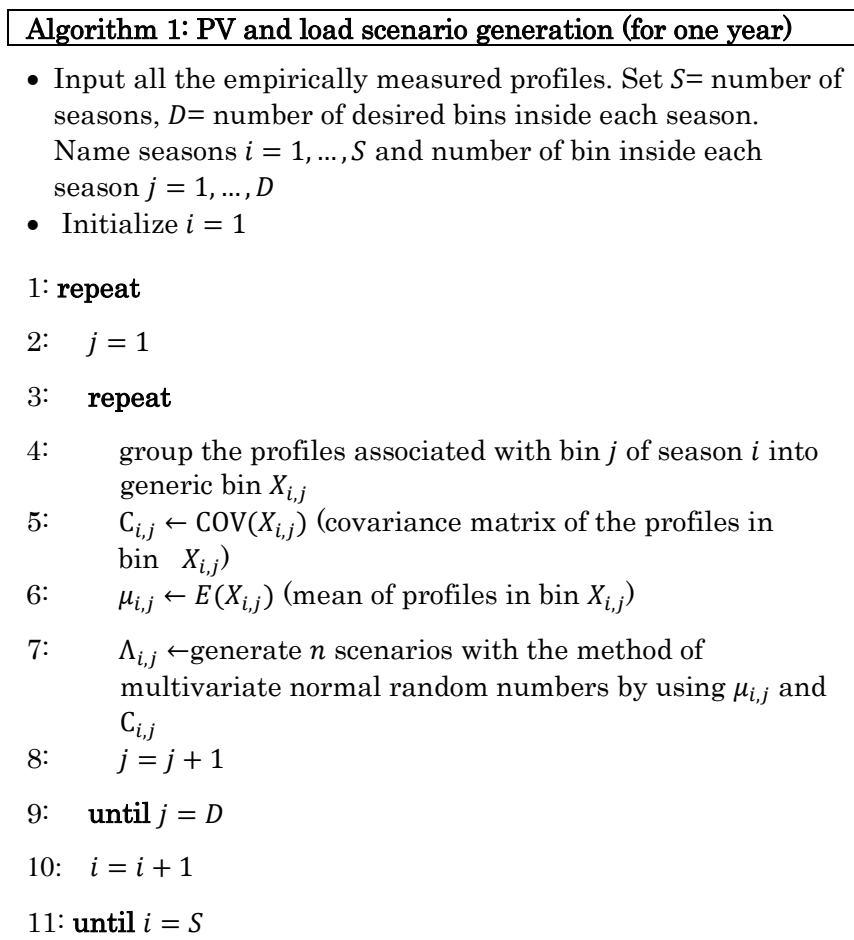

\section{Algorithm 2: Scenario reduction}

- Input all the scenarios associated with load, PV, and energy price from the algorithm 1 . Set the desired Euclidean distance between the final scenarios $\left(\Delta^{m}\right)$.

- Create the cluster tree including load, PV, and energy scenarios. $\mathcal{N}=$ number of scenarios associated with the scenario tree.

- Initialize $i=1$

1: repeat

2: $\quad j=1$

2: repeat

3: $\quad \Delta_{i, j} \leftarrow$ Euclidean distance bnetween time series $i$ and $j$

4: $\quad j=j+1$

5: $\quad$ until $j=\mathcal{N}$

6: $\quad i=i+1$

7: until $i=\mathcal{N}$

- Create the binary hierarchical cluster tree of scenarios using $\Delta$

- Create clusters of scenarios (criteria $\rightarrow \Delta^{m}$ as the maximum Euclidean distance)

- Choose one scenarios from each bin using K-Medoids 
switching, as we do not do switching frequently. Note that our working hypothesis is to have one configuration for each season. The ESS losses are neglected at this stage. We solve OPF, using linear DistFlow (see Section III.C) in the master problem to choose the optimal and feasible network configuration for each season (i.e., satisfying the static grid constraints composed by the nodal voltage-magnitude and lines’ ampacity limits). The network configuration is decided independently for each season and is identical for all the days within the season. We consider a multi-period OPF in the master problem accounting for the potential impacts of ESSs on the network configuration.

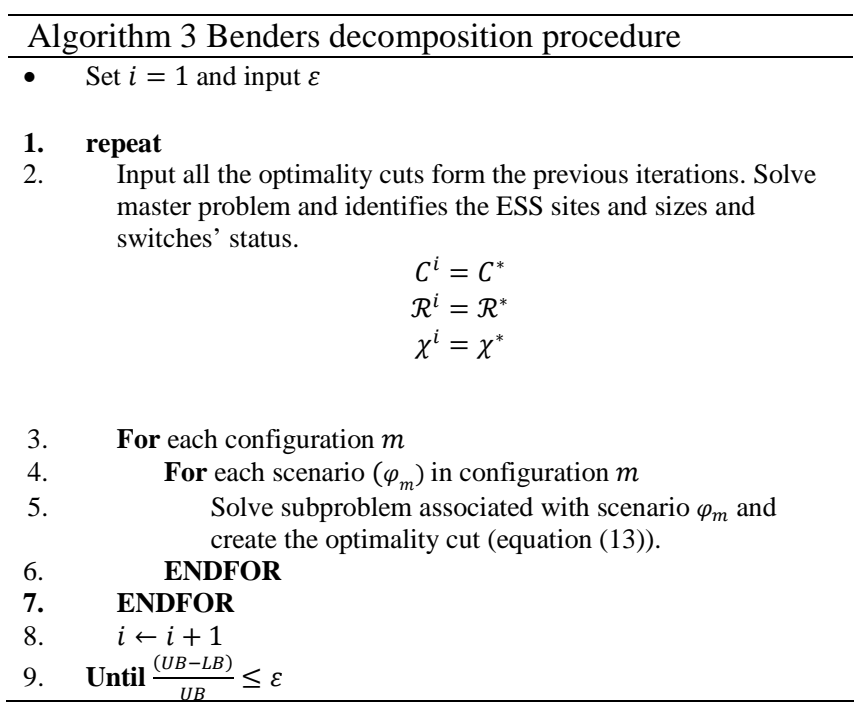

The determined ESS sites and sizes are fixed for the subproblems. In each iteration of subproblems, a set of constraints are added to the master problem representing the value of the identified ESS sites and sizes in the previous iteration. These constraints are formed based on the Lagrange multipliers associated with the fixed capacity constraint of the ESS units in the subproblems. The formulation of the Benders cut are explained in the next section.

The Master problem is formulated in (12). The constraints of the optimization problem are (i) geographical (i.e., the buses that cannot host ESS) and budget constraints for ESSs installation (ii) the radiality ones, and (iii) the constraints related to the seasonal network reconfiguration using DistFlow equations with inclusion of transverse parameters (see section III.C).

$$
\begin{gathered}
\operatorname{minimize}_{z, \zeta, \alpha, u, \Xi}: \mathcal{C}^{i}+\mathcal{C}^{s} \\
\mathcal{C}^{i}=\sum_{n \in \mathcal{N}}\left(C_{n}^{C} C_{n}+C_{n}^{\mathcal{R}} \mathcal{R}_{n}+C_{n}^{f} u_{n}\right) \\
+\sum_{t \in \mathcal{T}} \sum_{s \in \mathcal{S}} \sum_{(k, l) \in \mathcal{L}}\left(r _ { k l } \left(\left(P_{k l, s}(t)\right)^{2}\right.\right. \\
+\left(P_{l k, s}(t)\right)^{2}+\left(Q_{k l, s}(t)\right)^{2} \\
\left.\left.+\left(Q_{l k, s}(t)\right)^{2}\right)\right) \\
\mathcal{C}^{s}=\sum_{s \in \mathcal{S}} \sum_{\varphi \in \Upsilon_{s}} z_{s, \varphi}
\end{gathered}
$$

$$
\begin{gathered}
\Omega\left(\zeta_{s}(t), \theta_{s}(t)\right) \geq 0, \forall s \in \mathcal{S}, \forall t \in \mathcal{T} \\
\Psi\left(\alpha_{s}(t), \beta_{s}(t)\right) \geq 0, \forall s \in \mathcal{S}, \forall t \in \mathcal{T} \\
\sum_{n \in \mathcal{N}}\left(C_{n}^{C} C_{n}+C_{n}^{\mathcal{R}} \mathcal{R}_{n}+C_{n}^{f} u_{n}\right) \leq \mathcal{F} \\
C_{n} \leq u_{n} C_{n}^{\max }, \forall n \in \mathcal{N} \\
\mathcal{R}_{n} \leq u_{n} \mathcal{R}_{n}^{\max }, \forall n \in \mathcal{N} \\
Z_{s, \varphi} \geq \underline{Z}_{s, \varphi}, \forall s \in \mathcal{S}, \forall \varphi \in \Upsilon_{\mathrm{s}} \\
Z_{s, \varphi} \geq \Xi_{s, \varphi}^{i}, \forall s \in \mathcal{S}, \forall \varphi \in \Upsilon_{\mathrm{s}}, \forall i \in \mathcal{J} \\
u_{n} \in\{0,1\}
\end{gathered}
$$

where $C_{n}^{C}$ and $C_{n}^{\mathcal{R}}$ are the unit prices for energy reservoir $\left(C_{n}\right)$, and power rating $\left(\mathcal{R}_{n}\right)$ of ESSs, respectively. $C_{n}^{f}$ is associated with the fixed installation cost of the ESSs, and $u_{n}$ is the binary variable associated with the presence of an ESS on bus $n$. $n \in \mathcal{N}$ is the index of buses, $t \in \mathcal{T}$ is the index of time, $i \in$ $\mathcal{J}$ is the index of benders iterations, $s \in \mathcal{S}$ is the index of seasons (note that the network configuration is identical for all the scenarios inside each season), $\varphi \in \Upsilon_{\mathrm{s}}$ is the set of scenarios associated with season $m . \mathcal{F}$ is the total budget for ESSs installation, $Z_{s, \varphi}$ is a set of positive variables introduced to represent the subproblems cost, $\underline{Z}_{s, \varphi}$ represents the lower bound of the solution associated to each subproblem scenario, and $\Xi_{s, \varphi}^{i}$ represents the benders cuts from the subproblems (see equation (14)). The $\Theta(\Omega, \theta)$ and $\Psi(\alpha, \beta)$ represent the reconfiguration (see (10)) and ESS (see (7)) models, respectively. $\zeta$ and $\alpha$ are the set of variables for modelling the ADN reconfiguration and ESS installation and operation constraints, respectively. The decision variables are the ESS sites and sizes, ESSs active and reactive output, and network configuration for each scenario.

The objective includes: (i) the investment cost, composed by three terms, a) fixed cost $\left(C_{n}^{f} u_{n}\right)$, b) power rating cost $\left(C_{n}^{\mathcal{R}} \mathcal{R}_{n}\right)$ and c) energy reservoir cost $\left(C_{n}^{f} C_{n}\right)$; (ii) operation cost represented by the Benders cuts form the subproblems and (iii) total resistive losses (recall that $P_{l k}+j Q_{l k}$ is the complex power flow from bus $k$ to $l$ ( $l$ to $k$ ) after the shunt element of the line).

Equations (12.d) and (12.e) represent the set of ADN reconfiguration and ESSs constraints for all the scenarios and time steps. The budget limit is shown in the equation (12.f). The constraints (12.g) and (12.h) show the maximum capacity (power rating and energy) of ESS that can be installed on each particular bus. Equations (12.i) and (12.j) show the benders cuts.

\section{B. Subproblems}

For each season (configuration), a set of scenarios characterized by load, PV, and price variations assesses the operational benefits of the identified ESS sites and sizes. Each subproblem is a multi-period OPF with time-step discretization of 15 minutes. The objective is to decrease the nodal voltage-magnitude deviations, lines’ congestion and 
cost of supplied energy. The subproblem formulation is represented in equations (13). We remove the sub-script $s$ and $\varphi$, associated with the senarios, for notational conveniences. Note that our working hypothesis do not allow for PV curtailment. However, one can easily incorporate it in the problem using a new set of variables for PV curtailment.

$$
\begin{gathered}
\underset{\gamma, \alpha, \psi^{v}, \psi^{l}}{\operatorname{minimize}}: \mathcal{C}_{s, \varphi}=\sum_{t \in \mathcal{T}}\left[\sum_{l \in \mathcal{L}}(\overbrace{\mathcal{W}_{v} \psi_{l}^{v}(t)}^{1}+\overbrace{\mathcal{W}_{I} \psi_{l}^{I}(t)}^{2})\right. \\
+\overbrace{P_{1}^{t}(t) \xi(t) \Delta t}^{3}] \\
\psi_{l}^{v} \geq 0, \quad \forall l \in \mathcal{L}, \forall t \in \mathcal{T} \\
\psi_{l}^{v} \geq \underline{v}-v_{l}(t), \quad \forall l \in \mathcal{L}, \forall t \in \mathcal{T} \\
\psi_{l}^{v} \geq \hat{v}_{l}(t)-\bar{v}_{,} \quad \forall l \in \mathcal{L}, \forall t \in \mathcal{T} \\
\psi_{l}^{l} \geq 0, \quad \forall l \in \mathcal{L}, \forall t \in \mathcal{T} \\
\psi_{l}^{l} \geq \hat{I}_{l}^{t}(t)-\bar{I}_{l}, \quad \forall l \in \mathcal{L}, \forall t \in \mathcal{T} \\
\psi_{l}^{l} \geq \hat{I}_{l}^{b}(t)-\bar{I}_{l}, \quad \forall l \in \mathcal{L}, \forall t \in \mathcal{T} \\
\Theta(\gamma(t), \tau(t)) \geq 0, \quad \forall t \in \mathcal{T} \\
\Psi(\alpha(t), \beta(t)) \geq 0, \quad \forall t \in \mathcal{T} \\
C_{n}=C_{n}^{*}: \quad \mu_{n}, \forall n \in \mathcal{N} \\
\mathcal{R}_{n}=\mathcal{R}_{n}^{*}: \lambda_{n}, \forall n \in \mathcal{N}
\end{gathered}
$$

where $\mathcal{W}_{v}$, and $\mathcal{W}_{I}$ are the weighting coefficients associated with the voltage deviation minimization and congestion management (assuming that the energy supply cost weight is equal to 1). $P_{1}^{t}$ is the active power flow from the external transmission network to the grid (the active power flow at the substation transformer). $\hat{I}_{l}^{t}$ and $\hat{I}_{l}^{b}$ are upper bounds for the square of current flow at both ends of the line $l$ (see (4-d) and (4-c)). $v_{l}$ and $\hat{v}_{l}$ are the square of nodal voltage-magnitude of bus $l$ and its relevant auxiliary variable (see equations (1.b) and (3.b)). $\bar{v}$ and $\underline{v}$ are the maximum and minimum voltagemagnitude square beyond which nodal voltage-magnitude deviations are minimized. $\bar{I}_{l}$ is the square of line $l$ currentflow beyond which its current flow is minimized. $\mu_{n}$ and $\lambda_{n}$ are Lagrange multipliers of the fixed reservoir capacity and power rating of the ESS units. $\xi$ is the energy price, $\varphi \in \Upsilon_{S}$ is the index of scenarios, $t \in \mathcal{T}$ is the index of time steps, $n \in$ $\mathcal{N}$ is the index of buses, $l$ is the index of lines and the buses other than the slack bus, $C_{n}^{*}$ and $\mathcal{R}_{n}^{*}$ are parameters and are the identified optimal solution of master problem. The $\Theta(\gamma, \tau)$ and $\Psi(\alpha, \beta)$ represent the OPF (see (5)) and ESS (see (7)) models, respectively.

The first term in the objective function minimizes the nodal voltage-magnitude deviations. Under-voltages and overvoltages are minimized with respect to $\underline{v}$ and $\bar{v}$, respectively. Over-voltages are minimized using the auxiliary variable of voltage-magnitude square (recall this auxiliary variables are upper bounds for the voltage-magnitudes square). Similarly in the second term, the square of lines' current-flow (at both ends of the lines) above the maximum threshold, $\bar{I}_{l}$, are minimized using the auxiliary variables for the lines' currentflow square. The last term minimizes the energy supply cost with respect to the hourly energy price $(\xi)$.
The capacity of the ESSs power rating and energy reservoirs are fixed to the values obtained from the master problem in equations (13.j) and (13.k). The duals of these constraints are used to form the appropriate cuts as shown in (14) for the master problem. A Notation with ${ }^{*}$ represents a parameter fixed from the optimal solution of the previous iteration. $\pi_{s, \varphi}$ is the probability of the scenario $\varphi$ of $\Upsilon_{s}, \sigma$ is the annula interest rate, and $y$ is the index of the year after the installation of ESSs.

$$
\begin{aligned}
\Xi_{s, \varphi}^{i}=\frac{\pi_{s, \varphi}}{(1-\sigma)^{(1-y)}} & {\left[\mathcal{C}_{s, \varphi}^{*}\right.} \\
& -\sum_{n \in \mathcal{N}}\left(\hat{\mu}_{n, s, \varphi}\left(C_{n}-C_{n}^{*}\right)\right. \\
& \left.\left.+\hat{\lambda}_{n, s, \varphi}\left(\mathcal{R}_{n}-\mathcal{R}_{n}^{*}\right)\right)\right]
\end{aligned}
$$

\section{Simulation RESUltS}

This Section shows the effectiveness of the proposed methodology using two case studies. For the sake of clarity, the first one is a "toy example" composed of six buses and six lines. The second one is the standard 70-bus test case study. We use the second case to show the scalability of the proposed method.

Note that all the conditions stated in [7] hold for the two grids used in this paper. We check the conditions for each season, using the maximum and minimum levels of consumption and production, separately and they hold for all four seasons. This ensures the exactness of the relaxation used in AR-OPF. Furthermore, we have performed an ex-post load flow analysis regarding the exactness of the relaxation used in AR-OPF for all the simulated scenarios and never observed inexactness.

The price scenarios are extracted from EPEX price profiles in 2012, the load and PV profiles are coming from measurement in a primary substation in a region located in north of Italy and a region in south of Switzerland, respectively.

\section{A. Simple 6 lines, 6 nodes grid}

For the sake of reproducibility of the results, we have created a hypothetical small case study composed by 6 lines. The single line diagram of this network is shown in Fig. 3. The power, energy, and voltage base values are $1 \mathrm{MW}, 1$ MWh, and $4.6 \mathrm{kV}$, respectively. The resistance, reactance, and susceptance of the lines are 0.2328 p.u., 0.279 p.u., and 0.01 p.u., respectively. Note that all the lines have the same impedance and susceptance. The ESS installation cost parameters are $200 \$ / \mathrm{kWh}, 40 \$ / \mathrm{kVA}$, and 20000 \$/per site, for energy reservoir, power rating, and fixed cost, respectively. We have considered two seasons for the master problem, and 2 scenarios for the subproblem. The daily profile in the master problem has 12 time steps, whereas the subproblem profiles have 24 time steps. The Battery can perform 20,000 cycles; thus, with 20 years calendar lifetime $N_{c}$ is equal 2.7. The operation cost of the two scenarios are scaled up to 20 years. The data associated with the scenarios are provided in Table A.1 in Appendix I. The weighting coefficient of each element of the objective function is as follows: (i) $W_{E}=1$ (ESS investment cosst as well as the energy supply cost), (ii) $W_{v}=20$ (nodal voltage magnitudes 
deviations), and (iii) $W_{l}=20$ (lines congestions). Both the master and subproblems are solved using the solver Gurobi [32] via the MATLAB interface YALMIP [33] on a desktop PC with Intel Xeneon CPU $3.2 \mathrm{GHz}$ and 16 GB RAM. The resistance of the ESSs, upper voltage-magnitude limit, and lower voltage-magnitude limit are considered 0.01 p.u., 1.05 p.u. and 0.92 p.u., respectively. Table I shows the peak active and reactive load as well as the installed PV capacity of each bus. Table II illustrates the current-flow limit of the lines. The identified optimal ESS sites and sizes are shown in Table III.

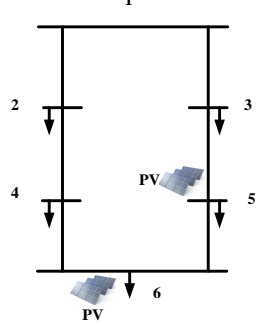

Fig. 3: Hypothetical 6 lines grid

Table I: Nodal peak active and reactive load and PV capacity

\begin{tabular}{|c|c|c|c|c|c|c|}
\hline Bus\# & $\begin{array}{l}\text { Peak active } \\
\text { load }(\mathrm{kW})\end{array}$ & & $\begin{array}{r}\text { Peak reactiv } \\
(\mathrm{kVar}\end{array}$ & e load & $\begin{array}{r}\text { PV c } \\
{[\mathrm{k}}\end{array}$ & $\begin{array}{l}\text { pacity } \\
\text { W] }\end{array}$ \\
\hline 2 & 27.73 & & 4.44 & & & 5 \\
\hline 3 & 27.73 & & 4.44 & & & 5 \\
\hline 4 & 356.61 & & 33.28 & & & 5 \\
\hline 5 & 85.58 & & 9.99 & & & .94 \\
\hline 6 & 362.95 & & 29.96 & & & .18 \\
\hline \multicolumn{7}{|c|}{ Table II: lines' current-flow limit } \\
\hline Line & $(1,2)$ & $(1,3)$ & $(2,4)$ & $(3,5)$ & $(4,6)$ & $(5,6)$ \\
\hline $\begin{array}{c}\text { Current flow } \\
\text { limit [p.u] }\end{array}$ & 0.1428 & 0.179 & 0.1325 & 0.1687 & 0.179 & 0.1366 \\
\hline
\end{tabular}

Table III: Identified optimal ESS sites and sizes for 6 bus grid

\begin{tabular}{ccc}
\hline \hline Bus \# & $\begin{array}{c}\text { Power rating } \\
(\mathrm{kVA})\end{array}$ & $\begin{array}{c}\text { Energy reservoir } \\
(\mathrm{kWh})\end{array}$ \\
\hline 4 & 72.35 & 175.58 \\
\hline 5 & 52.94 & 2.66 \\
\hline 6 & 114.16 & 294.89 \\
\hline
\end{tabular}

Table III shows that three buses are selected for ESS installation. The total ESS capacity installation is equal to $239.45 \mathrm{~kW}$ and $473.14 \mathrm{kWh}$.

For the identified optimal solution, the switch between the buses \#5 and \#6 is open for the season 2, whereas the switch between buses $4 \#$ and $6 \#$ is open in season 1 .

Fig. 4 depicts the Cumulative Distribution Functions (CDFs) of the nodal voltage-magnitudes related to two cases, (i) with proposed procedure for optimal siting and sizing of ESSs, (ii) without ESS (base case) ${ }^{4}$. One can easily observe that the first case, optimal siting and sizing of ESSs taking into account network reconfiguration, has better voltage profile. In particular, the voltage magnitudes are well kept in the desired operation region ( $\pm 3 \%$ deviation). Furthermore it can be seen that the voltage magnitudes below 0.97 p.u. and larger than 1.03 p.u. are completely removed.

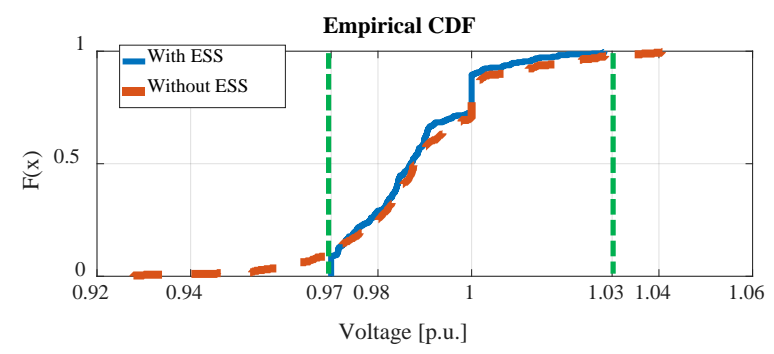

Fig. 4: CDF of nodal voltage-magnitude for (i) base case without ESS and (ii) optimal ESS siting and sizing with proposed procedure

Table IV: Improvement in the objective function goals for the 6 bus network

\begin{tabular}{c|c|c}
\hline \hline & $\begin{array}{c}\text { Energy cost } \\
\text { imported from } \\
\text { the external grid } \\
\text { [CHF] }\end{array}$ & $\begin{array}{c}\text { Lines with congestion } \\
\text { (loading above 80\% } \\
\text { of nominal rating) }\end{array}$ \\
\hline $\begin{array}{c}\text { with optimal ESS placement } \\
\text { considering network configuration }\end{array}$ & $398,864.7$ & - \\
\hline without ESS & 412,366 & $\begin{array}{c}(1,2),(1,3), \\
(2,4),(3,5),(5,6),(4,6)\end{array}$ \\
\hline \hline
\end{tabular}

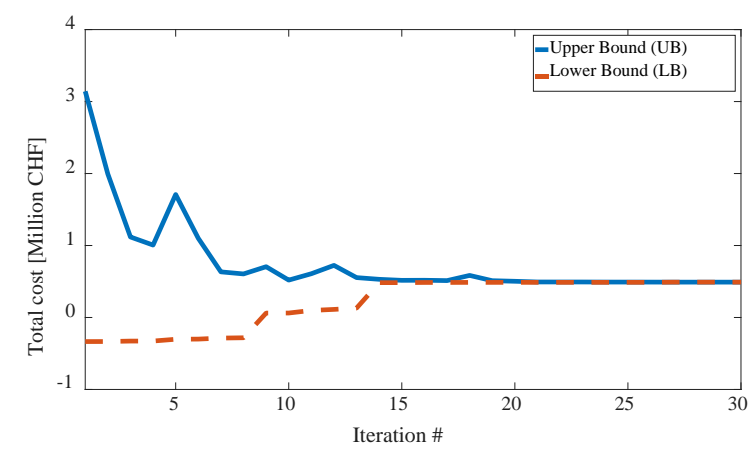

Fig. 5: convergence of the proposed Benders decomposition

Table V: the average computation time for 6-bus network

\begin{tabular}{c|c|c}
\hline \hline & Master Problem & Subproblem \\
\hline Average computation time [s] & 1.82 & 0.53 \\
\hline \hline
\end{tabular}

Table IV shows the improvements of the other elements of the objective function. One can observe that the lines' congestions are completely removed with the use of optimally sited and sized ESSs. Additionally, the energy supply cost has been decreased by $3.27 \%$. Note that the energy cost in Table IV represents the energy supply cost for the whole simulation period, and then scaled to 20 years.

The convergence of the proposed decomposition procedure with Benders method is shown in Fig. 5. In particular, the process converged after 27 iterations (the stopping criteria is $\left.\frac{|U B-L B|}{U B} \leq 0.001\right)$. Table $\mathrm{V}$ shows the average computation time associated with the master problem and subproblems.

Note that the five conditions introduced in [7] holds for identified radial configurations of this grid for both scenarios (these conditions depends on the lines parameters and maximum nodal injections and absorptions; thus can be checked ex-ante). 


\section{1) Comparison with the case where network reconfiguration is not considered}

In this section, we present a comparison with the case where the network reconfiguration is not considered. We used the same parameters of the previous simulations. The only difference is that we consider a single network configuration for the grid. To reach the same level of voltage and line current quality as the previous case, $568.23 \mathrm{~kW}$ ESS power rating and 2.08 MWh ESS energy reservoir is required (in the previous case where we accounted for the reconfiguration, the required power/energy ratings are $239.45 \mathrm{~kW}$ and 473.14 $\mathrm{kWh}$ ). The total cost, (investment cost + operation cost) has also increased of $36.3 \%$ compared to the previous case.

\section{B. 70-bus standard network}

The modified 70-bus network of [28] (shown in Fig. 6) is selected to verify the effectiveness of the proposed planning procedure with respect to a standard network. This test case is a hypothetical $11 \mathrm{kV}$ radial distribution system that has 1 substations, 2 feeders, 69 nodes, 12 tile-lines, peak load of 1.3 MW, PV capacity of $1.2 \mathrm{MW}$, and 76 branches. We have assumed a battery-life time of 20 years and considered 4 seasonal configuration in the master problem and 60 scenarios each five years for the subproblem (the scenarios are created using the scenarios generation/reduction method presented in section (III.D)).

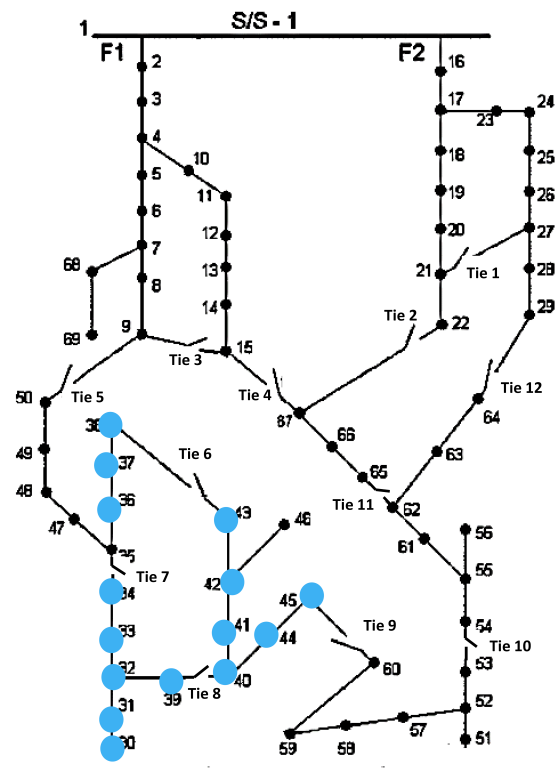

Fig. 6: 70-bus network (the PV sites are shown in blue) [29]

Both the master and subproblems are solved using the solver Gurobi [31] via the MATLAB interface YALMIP [32].

We have assumed that the DNO has constraints on the ESS installation sites. To represent this constraint, we assumed a list of 28 candidate buses for installing ESSs (see Table VI) . The ESSs installation costs are considered to be $300 \$ / \mathrm{kWh}$, $100 \$ / \mathrm{kVA}$, and 20000 \$/per site. Other simulation parameters are provided in Table VII.

The identified optimal sites and sizes of the ESSs units are provided in Table IX. In particular, five buses are selected for installing ESS units. Table X shows the switches statue for the two identified network configurations. Note that the network configurations for summer, spring, and fall are identical.

In the following, we present the comparison between three cases: (i) base case without ESS (optimal network reconfiguration is considered), (ii) optimally located ESS without network reconfiguration, and (iii) optimally located ESSs considering network reconfiguration. Note that we used the same parameters for all the three cases.

Table XI shows total amount of required ESS power rating and energy reservoir for cases (ii) and (iii). One can observe that considering the network reconfiguration significantly decreased the total ESS size. In particular, total energy reservoir capacity and power rating are cut off by $26 \%$ and $42 \%$, respectively.

Fig. 7 shows the Cumulative Distribution Functions (CDFs) of the nodal voltage magnitudes related to three cases. These results clearly show that optimal siting and sizing of ESSs taking into account network reconfiguration, has the best voltage profile. In particular, it can be observed that the voltage magnitudes are well kept within the desired operating region (0.96 p.u. -1.02 p.u.). Furthermore it can be seen that the voltage magnitudes below 0.94 p.u. and larger than 1.02 p.u. are completely removed. It is interesting to observe that although the case without network reconfiguration has larger ESS capacities with respect to the case with reconfiguration (see Table XI), it has slightly worse voltage-magnitude profile than the case with network reconfiguration.

Improvements in the other objectives are presented in Table XII. Particularly, it can be observed that with optimally located ESS units taking into account network reconfiguration the number of lines with loading above $80 \%$ are decreased from 46 to 3 . Furthermore, the energy supply cost is decreased by $15.1 \%$ with respect to the base case. It is also interesting to observe that the case without network reconfiguration has more lines with loading above $80 \%$ with respect the proposed procedure, even though it has ESSs with more capacity.

Table VI: Candidate buses for installing ESSs

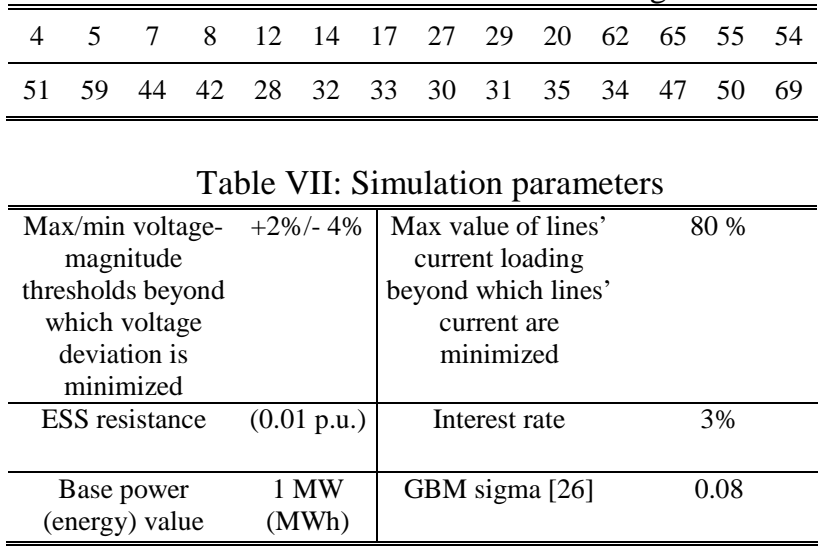

Table IX: Identified optimal ESS sites and sizes ${ }^{5}$ (PR: Power rating, ER: Energy Reservoir)

\begin{tabular}{c|c|c|c|c|c|c}
\hline \hline with network & Bus\# & 32 & 34 & 42 & 54 & 65 \\
\cline { 2 - 7 } $\begin{array}{c}\text { reconfiguration } \\
\text { possibility }\end{array}$ & PR (kVA) & 138 & 62 & 53 & 162 & 21 \\
\cline { 2 - 7 } & ER (kWh) & 315 & 280 & 194 & 441 & 57 \\
\hline \hline
\end{tabular}


Table X: Closed switches for the identified configurations

\begin{tabular}{c|c|c|c|c|c|c}
\hline \hline & \multicolumn{5}{|c}{ Closed switch (tie-line) \# } \\
\hline winter & 2 & 5 & 6 & 7 & 10 & 11 \\
\hline Summer, spring, and fall & 2 & 5 & 7 & 9 & 10 & 11 \\
\hline \hline & \multicolumn{5}{c}{$\begin{array}{c}\text { Energy reservoir } \\
\text { Table XI: Total identified optimal ESS capacities with and without } \\
\text { considering network reconfigution }\end{array}$} & $\begin{array}{c}\text { Power rating } \\
\text { (kVA) }\end{array}$ \\
\hline \hline & 1.287 & 436 \\
\hline $\begin{array}{c}\text { Optimal ESS siting and sizing } \\
\text { with reconfiguration }\end{array}$ & 1.752 & 752 \\
\hline $\begin{array}{c}\text { Optimal ESS siting and sizing } \\
\text { without reconfiguration }\end{array}$ & &
\end{tabular}

Table XII: improvement in the objective function goals

\begin{tabular}{c|c|c}
\hline \hline & $\begin{array}{c}\text { Total operation cost for } \\
\text { the whole period (20 } \\
\text { years)) [CHF] }\end{array}$ & $\begin{array}{c}\text { Number of lines with } \\
\text { congestion (loading } \\
\text { above 80\% of the line } \\
\text { rating) }\end{array}$ \\
\hline $\begin{array}{c}\text { with optimal ESS } \\
\text { placement considering }\end{array}$ & $1,631,580$ & 3 \\
$\begin{array}{c}\text { network configuration } \\
\text { (Total ESS capacities : }\end{array}$ & \\
$\begin{array}{c}\text { 1.287 MWh, 436 KVA) } \\
\text { without ESS (base } \\
\text { case) }\end{array}$ & $1,922,980$ & 46 \\
\hline $\begin{array}{c}\text { with optimal ESS } \\
\text { placement considering } \\
\text { network configuration } \\
\text { (Total ESS capacities : }\end{array}$ & $1,640,783$ & 14 \\
\hline 1.752 MWh, 752 KVA) & & \\
\hline
\end{tabular}

Note that the weighting coefficient of each element of the objective function is as follows: (i) $W_{E}=1$ (ESS investment costs as well as the energy supply cost), (ii) $W_{v}=20$ (nodal voltage-magnitudes' deviations), and (iii) $W_{l}=40$ (lines' congestion).

The proposed decomposition procedure based on Benders method have converged after 38 iterations (the stopping criteria is $\left.\frac{|U B-L B|}{U B} \leq 0.1 \%\right)$. The average computation time associated with master and subproblems are shown in Table XIII.

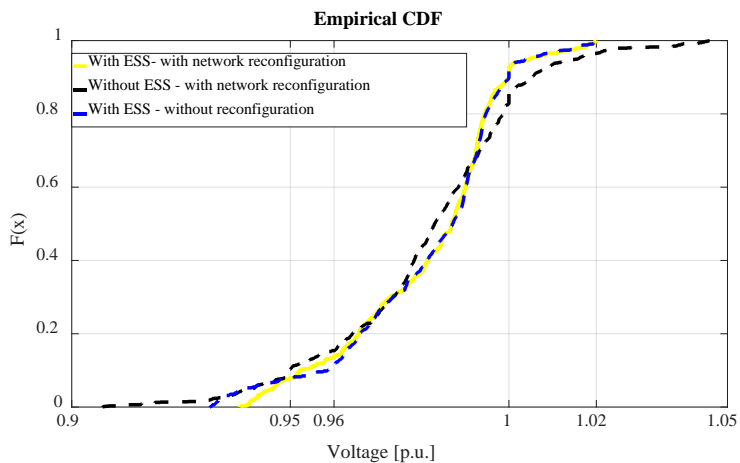

Fig. 7: Nodal voltage magnitudes CDF, (i) with optimal placement of ESSs embedding network reconfiguration, (ii) with optimal placement of ESSs, and (iii) without ESSs

Table XIII: the average computation time associated with master and subproblems of 70-bus test case study

\begin{tabular}{c|c|c}
\hline \hline & Master Problem & Subproblem \\
\hline Average computation time & $1.3[\mathrm{~min}]$ & $0.73[\mathrm{~s}]$ \\
\hline \hline
\end{tabular}

Finally, note that the 5 conditions introduced in [7] holds for identified radial configurations of this grid.

\section{1) Sensitivity analysis with respect to the weighting terms in the objective function}

The results of multi-objective optimizations are sensitive to the weighting coefficients in the objective function, e.g., investment cost, voltage deviation minimization and lines' congestion management in (11). In Table XIV, we provide a sensitivity analysis with respect to the variation of the weighting coefficients of the objective function.

As it one can see from Table XIV, 2.3 MWh and 0.863 MVA of energy storage are required to completely remove the lines' loading above $80 \%$ in all the scenarios for all time steps. Furthermore, the required amount of ESS capacity decrease as one decrease the weighting coefficients of the lines congestion minimization and nodal voltage-magnitude deviations. For example with $1.01 \mathrm{MWh} / 0.435$ MVA ESS the number of lines with loading above $80 \%$ increase from 3 to 20 .

Table XIV: ESS capacities with different weights for voltage-magnitude and lines' flow minimization

\begin{tabular}{ccccc}
\hline \hline & $\begin{array}{c}\text { Weighting } \\
\text { coefficients for } \\
\text { voltage and } \\
\text { current } \\
\left(W_{v}-W_{l}\right)\end{array}$ & $\begin{array}{c}\text { ESS capacities } \\
\text { (MWh - MVA) }\end{array}$ & $\begin{array}{c}\text { Number of } \\
\text { lines with } \\
\text { loading above } \\
8 \%\end{array}$ & $\begin{array}{c}\text { Minimum and } \\
\text { maximum voltage } \\
\text { magnitudes }\end{array}$ \\
\hline Case I & $(50-50)$ & $2.3-0.863$ & 0 & $0.965-1.097$ \\
\hline Case II & $(30-45)$ & $1.87-0.650$ & 1 & $0.931-1.097$ \\
\hline Case III & $(20-40)$ & $1.287-0.436$ & 3 & $0.93-1.02$ \\
\hline Case IV & $(5-15)$ & $1.01-0.435$ & 20 & $0.912-1.02$ \\
\hline \hline
\end{tabular}

\section{CONCLUSION}

The paper proposes a process for optimal ESSs allocation into Active Distribution Networks (ADNs) embedding grid reconfiguration. In addition to the investment cost of ESSs, the capability of ESSs to support the network in terms of: (i) network voltage deviations, (ii) feeders congestion, (iii) network losses, (iv) and cost of supplying loads (from external grid or local producers) has been accounted for to find their best locations and sizes. To solve this problem, we have appropriately adapted a recently proposed convex OPF model integrated with a suitable set of constraints to exploit network reconfiguration capability.

Benders decomposition is used to break down the targeted optimization problem enabling its fast/accurate solution. This peculiarity has allowed the analysis of multiple scenarios, generated by a suitable process. It accounts for the stochastic behavior of both loads and renewables together with their evolution in terms of growth and price changes along the ESS lifetime.

The obtained results have shown the capabilities of the proposed methods to optimally allocate ESSs to: (i) largely improve the quality of supply of the ADNs in terms of mitigating voltage deviations, eliminating line congestions together with (ii) minimizing the total cost of locally used electricity and investment cost for ESSs installation. It is possible to conclude that optimally allocated ESSs can 
represent a valid solution for ADN operators that do not want to deploy massive DG controls. This opportunity will potentially postpone large control infrastructure deployment as well as grid infrastructure reinforcement.

\section{REFERENCES}

[1] P. Mercier, R. Cherkaoui, A. Oudalov, "Optimizing a battery energy storage system for frequency control application in an isolated power system," IEEE Trans. Power Syst., vol. 24, no. 3, pp. 1469 -1477, Aug. 2009.

[2] G. Celli, E. Ghiani, S. Mocci, F. Pilo "A multi-objective evolutionary algorithm for the sizing and siting of distributed generation," IEEE Trans. on Power Systems, vol. 20, no. 2, pp. 750-757, May 2005.

[3] M. Nick, R. Cherkaoui, and M. Paolone, "Optimal allocation of dispersed energy storage systems in active distribution networks for energy balance and grid support”, IEEE Trans. Power Syst., vol. 29, no. 5, pp. 2300-2310, Sept. 2014.

[4] S. Nykamp, M. G. C. Bosman, A. Molderink, J. L. Hurink and G. J. M. Smit, "Value of Storage in Distribution Grids-Competition or Cooperation of Stakeholders?" IEEE Trans. Smart Grid, vol. 4, pp. 1361-1370, Sept. 2013.

[5] L. Gan, N. Li, U. Topcu, and S. Low, "On the Exactness of Convex Relaxation for Optimal Power Flow in Tree Networks," 2012 IEEE Annual conference o decision and control, pp. 465 - 471.

[6] G. Lingwen L. Na, U. Topcu, S. Low, "Exact Convex Relaxation of Optimal Power Flow in Radial Networks," IEEE Trans. Automatic Control, vol.60, no.1, pp.72,87, Jan. 2015.

[7] M. Nick, R. Cherkaoui, J.-Y. Le Boudec, and M. Paolone, “An Exact Convex Formulation of Optimal Power Flow in Radial Distribution Networks Including Transverse Components", arXiv preprint arXiv:1605.01964.

[8] A. J. Conejo, E. Castillo, R. Minguez, R. Garcia-Bertrand, "Decomposition techniques in mathematical programming: engineering and science applications," Springer Science \& Business Media, ch. 6, sec. 6.4, 2006

[9] Geoffrion, Arthur M. "Generalized benders decomposition." Journal of optimization theory and applications 10, no. 4 (1972): 237-260.

[10] F. You, I. E. Grossmann, "Multicut Benders decomposition algorithm for process supply chain planning under uncertainty", Annals of Operations Research, vol. 210, pp 191-211, 2013.

[11] P. S. Georgilakis and N. D. Hatziargyriou, "Optimal Distributed Generation Placement in Power Distribution Networks: Models, Methods, and Future Research," IEEE Transactions on Power Systems, vol. 28, pp. 3420-3428, 2013

[12] C. Mateo, J. Reneses, A. Rodriguez-Calvo, P. Frías and Á Sánchez, "Cost-benefit analysis of battery storage in medium-voltage distribution networks," IET Generation, Transmission \& Distribution, vol. 10 , pp. 815-821, 2182016.

[13] C. Chen, S. Duan, T. Cai, B. Liu, and G. Hu "Optimal allocation and economic analysis of energy storage system in microgrids," IEEE Trans. on Power Electronics, vol. 26, no. 10, pp. 2662-2773, Oct. 2011.

[14] M. Nick, M. Hohmman, R. Cherkaoui, and M. Paolone, "Optimal Location and Sizing of Distributed Storage Systems in Active Distribution Networks," 2013 IEEE PowerTech (POWERTECH), pp.16.

[15] C. Mateo, J. Reneses, A. Rodriguez-Calvo, P. Frías and Á Sánchez, "Cost-benefit analysis of battery storage in medium-voltage distribution networks," in IET Generation, Transmission \& Distribution, vol. 10, no. 3, pp. 815-821, 2182016.
[16] J. Xiao, Z. Zhang, L. Bai and H. Liang, "Determination of the optimal installation site and capacity of battery energy storage system in distribution network integrated with distributed generation," in IET Generation, Transmission \& Distribution, vol. 10, no. 3, pp. 601-607, 2 18, 2016.

[17] F. Abbasi and S. M. Hosseini, "Optimal DG allocation and sizing in presence of storage systems considering network configuration effects in distribution systems," IET Gen., Tran. \& Dist., vol. 10, pp. 617-624, 2016.

[18] G. Carpinelli, G. Celli, S. Mocci, F. Mottola, F. Pilo and D. Proto, "Optimal Integration of Distributed Energy Storage Devices in Smart Grids," in IEEE Transactions on Smart Grid, vol. 4, no. 2, pp. 985-995, June 2013.

[19] A. Motalleb, E. Reihani, R. Ghorbani, "Optimal placement and sizing of the storage supporting transmission and distribution networks", Renewable Energy, vol. 94, pp. 651-659, 2016.

[20] Y. Zheng, Y. D. Zhao, J. Feng, K Meng, J Qiu, and K. P. Wong. "Optimal Allocation of Energy Storage System for Risk Mitigation of DISCOs With High Renewable Penetrations”, IEEE Trans. Power Syst., vol. 29, pp. 212-220, 2014.

[21] S. W. Alnaser and L. F. Ochoa, "Optimal Sizing and Control of Energy Storage in Wind Power-Rich Distribution Networks," IEEE Trans. on Power Syst., vol. 31, pp. 2004-2013, May 2016.

[22] Q. Li, R. Ayyanar and V. Vittal, "Convex Optimization for DES Planning and Operation in Radial Distribution Systems with High Penetration of Photovoltaic Resources," in IEEE Trans. on Sust. Ener., vol. 7, no. 3, pp. 985-995, July 2016.

[23] M. Nick, R. Cherkaoui, M. Paolone, "Optimal siting and sizing of distributed energy storage systems via alternating direction method of multipliers" International Journal of Electrical Power \& Energy Systems, vol. 72, Pages 33-39, 2015.

[24] K. Christakou, D. C. Tomozei, J.Y. Le Boudec, and M. Paolone, “AC OPF in Radial Distribution Networks - Part I: On the Limits of the Branch Flow Convexification and the Alternating Direction Method of Multipliers” to appear on Electric Power Systems Research, 2016.

[25] E. Namor, D. Torregrossa, F. Sossan, R. Cherkaoui and M. Paolone, "Assessment of battery ageing and implementation of an ageing aware control strategy for a load leveling application of a lithium titanate battery energy storage system," 2016 IEEE 17th Workshop on Control and Modeling for Power Electronics (COMPEL), Trondheim, 2016, pp. 1-6.

[26] J. A. Taylor and F. S. Hover, "Convex Models of Distribution System Reconfiguration," in IEEE Trans. Power Syst., vol. 27, pp. 1407-1413, Aug. 2012

[27] M. E. Baran and F. F. Wu, "Optimal capacitor placement on radial distribution systems,” IEEE Trans. Power Delivery, vol. 4, no. 1, pp. 725-734, 1989.

[28] M. T. Barlow, "A diffusion model for electricity prices," Mathematical Finance, vol. 12, pp. 287-298, 2002.

[29] H.-S. Park and C.-H. Jun, "A simple and fast algorithm for K-medoids clustering," Expert Systems with Applications, vol. 36, pp. 3336-3341, $3 / / 2009$

[30] D. Das, "Reconfiguration of distribution system using fuzzy multiobjective approach,” Int. J. Electr. Power Energy Syst., vol. 28, no. 5, pp. 331-338, 2006.

[31] J. Löfberg, "YALMIP: A toolbox for modeling and optimization in MATLAB," in Proc. CACSD Conf., Taipei, Taiwan, 2004, pp. 284 289.

[32] "Gurobi Optimizer Reference Manual," Gurobi Optimization, Inc. , 2012 [Online]. Available: http://www.gurobi.com 


\section{APPENDIX I}

Table A.1: Time series associated with the load, PV, and energy price scenarios (for master problem profiles where we have time step length of 2 hours, we select the maximum value of the corresponding two hours)

\begin{tabular}{|c|c|c|c|c|c|c|c|c|c|c|c|c|}
\hline Time step (hour) & 1 & 2 & 3 & 4 & 5 & 6 & 7 & 8 & 9 & 10 & 11 & 12 \\
\hline $\begin{array}{l}\text { Total Load in per unit (base value } 1 \text { MVA) } \\
\text { (Scenario \#1) }\end{array}$ & 0.296 & 0.287 & 0.287 & 0.279 & 0.269 & 0.287 & 0.317 & 0.362 & 0.441 & 0.587 & 0.622 & 0.633 \\
\hline $\begin{array}{l}\text { Total Load in per unit (base value } 1 \text { MVA) } \\
\text { (Scenario \#2) }\end{array}$ & 0.312 & 0.299 & 0.293 & 0.293 & 0.282 & 0.281 & 0.291 & 0.298 & 0.312 & 0.324 & 0.336 & 0.343 \\
\hline Total PV in per unit (Scenario \#1) & 0.000 & 0.000 & 0.000 & 0.000 & 0.000 & 0.000 & 0.000 & 0.003 & 0.009 & 0.017 & 0.023 & 0.026 \\
\hline $\begin{array}{c}\text { Total PV in per unit of total peak PV } \\
\text { capacity (base value } 1 \mathrm{MW} \text { ) (Scenario \#2) }\end{array}$ & 0.000 & 0.000 & 0.000 & 0.000 & 0.007 & 0.056 & 0.186 & 0.367 & 0.528 & 0.653 & 0.720 & 0.740 \\
\hline Energy price (CHF/MWh) (Scenario \#1) & 29.10 & 26.33 & 24.30 & 23.45 & 25.84 & 33.55 & 42.43 & 46.77 & 47.66 & 46.51 & 45.94 & 43.56 \\
\hline Energy price (CHF/MWh) (Scenario \#2) & 24.41 & 22.16 & 19.97 & 19.94 & 23.11 & 28.27 & 34.41 & 37.27 & 37.69 & 38.02 & 38.52 & 36.84 \\
\hline Time step (hour) & 13 & 14 & 15 & 16 & 17 & 18 & 19 & 20 & 21 & 22 & 23 & 24 \\
\hline $\begin{array}{l}\text { Total Load in per unit (base value } 1 \text { MVA) } \\
\text { (Scenario \#1) }\end{array}$ & 0.634 & 0.472 & 0.430 & 0.470 & 0.647 & 0.785 & 0.861 & 0.843 & 0.567 & 0.466 & 0.384 & 0.336 \\
\hline $\begin{array}{l}\text { Total Load in per unit (base value } 1 \text { MVA) } \\
\text { (Scenario \#2) }\end{array}$ & 0.342 & 0.328 & 0.335 & 0.343 & 0.361 & 0.386 & 0.418 & 0.427 & 0.405 & 0.374 & 0.345 & 0.319 \\
\hline $\begin{array}{l}\text { Total PV in per unit (base value } 1 \mathrm{MW} \text { ) } \\
\text { (Scenario \#1) }\end{array}$ & 0.024 & 0.018 & 0.010 & 0.004 & 0.001 & 0.000 & 0.000 & 0.000 & 0.000 & 0.000 & 0.000 & 0.000 \\
\hline $\begin{array}{l}\text { Total PV in per unit (base value } 1 \mathrm{MW} \text { ) } \\
\text { (Scenario \#2) }\end{array}$ & 0.710 & 0.628 & 0.494 & 0.345 & 0.195 & 0.068 & 0.010 & 0.000 & 0.000 & 0.000 & 0.000 & 0.000 \\
\hline Energy price (CHF/MWh) (Scenario \#1) & 41.61 & 42.16 & 42.58 & 42.98 & 44.18 & 45.40 & 47.72 & 46.38 & 44.08 & 43.19 & 37.99 & 33.61 \\
\hline Energy price (CHF/MWh) (Scenario \#2) & 35.53 & 34.68 & 34.06 & 33.47 & 34.37 & 35.54 & 35.49 & 34.63 & 34.10 & 34.46 & 30.37 & 26.98 \\
\hline
\end{tabular}

